\title{
Crystal and Solution structures of Proliferating Cell Nuclear Antigen from Crenarchaeon Aeropyrum pernix
}

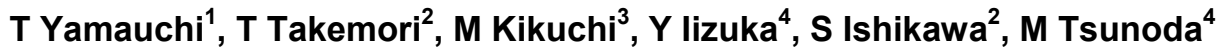 \\ ${ }^{1}$ Graduate School of Life Science and Technology, Iryo Sosei University, Japan, Iwaki, Fukushima, \\ ${ }^{2}$ Faculty of Pharmacy, Iryo Sosei University, Iwaki, Fukushima, ${ }^{3}$ Graduate School of Science and \\ Engineering, Iryo Sosei University, Iwaki, Fukushima, ${ }^{4}$ Graduate School of Life Science and \\ Technology, Iryo Sosei University, Iwaki, Fukushima \\ df2002@isu.ac.jp
}

Introduction Sliding clamps are ring-shaped proteins that encircle DNA and confer high processivity on DNA polymerases. In bacteria, the $\beta$-clamp protein forms a homodimer, whereas in eukaryotes or euryarchaeotes, proliferating cell nuclear antigen (PCNA) proteins form homotrimers. However, PCNA from Aeropyrum pernix (ApPCNA), a crenarchaeote species, forms a heterotrimer. The actual structure of ApPCNA-mediated sliding clamps and the mechanism by which they slide along DNA is unknown. The present study aimed to analyze the crystal and solution structure of the heterotrimeric ring of ApPCNA, examine its interaction with DNA and other proteins, and elucidate the mechanism of PCNA function. Previously, we have analyzed the crystal structure of ApPCNA1 from the APE_0162 gene.1)

Experimental procedures Each ApPCNA molecule, which constitutes a heterotrimer, was expressed using the Escherichia coli expression system. The proteins were purified using heat treatment, ammonium sulfate precipitation, and column chromatography. The purified proteins were crystallized using the vapor-diffusion method and the crystals were analyzed by Xray diffraction. The crystal structures of ApPCNA2 from the APE_0441.1 gene and ApPCNA3 from the APE_2182 gene were determined by the single-wavelength anomalous dispersion method using platinum. To verify the ring shape of ApPCNA2 in solution, the solution structure was analyzed using size-exclusion chromatography-small-angle X-ray scattering (SEC-SAXS). A mixture of ApPCNAs was analyzed by SEC-multi-angle light scattering for the presence of a complex, and the solution structure was analyzed by SEC-SAXS.

Results Crystals of ApPCNA2 were grown up to $0.1 \mathrm{~mm}$ and diffracted to 1.8 A resolution. ApPCNA2 crystallized as a tetramer. Two sets of dimers, linked through hydrogen bonds, joined back to back in an asymmetric unit. This structure also formed a tetrameric-ring. Crystals of ApPCNA3 diffracted to 1.9 A resolution. The structure formed a trimeric ring. The Cterminal interacted with the cleavage site, a possible PCNA-interacting peptide box (PIP-box) binding site, of the adjacent trimeric ring. The solution structure of the complex was similar to shape of the British Isles islands. ApPCNA2 and ApPCNA3 interacted in a similar manner as the PCNA rings of other organisms; however, ApPCNA1 was located such that it did not form a perfect ring-shaped structure. The scattering curves of the complex and those of the model edited trimeric ring were almost similar with minor differences.

Discussion The crystal structure of ApPCNA2 showed that it exists as a tetrameric-ring. PCNA is a trimeric ring. Although this molecule, which forms a trimer, is unstable as a tetramer, the crystal structure forms a tetrameric-ring. Hydrogen bonds present in neighboring molecule, from Thr175 to Glu179 and Glu109 to Ile114, form a $\beta$-sheet. This flexible interaction suggests that the protein could be forming tetrameric-ring-shaped structure. The crystal structure of ApPCNA3 shows that the C-terminal contacts the cleavage site surrounded by $\beta$-sheets, formed behind the $\alpha$-helices, and the inter-domain connecting loop between the neighboring trimeric-rings. Leu249 in the C-terminal is placed in the hydrophobic pocket formed by Met40, Leu47, Leu131, Pro228, Ala246, and Pro247 (Figure). Thus, this hydrophobic cleavage site may be a binding site for PIP-box motif containing proteins. The N-terminus of ApPCNA1 is approximately 10 residues longer than that of ApPCNA2 and ApPCNA3. This could be why the tripartite complex is not ring shaped. Moreover, Met16 is present downstream of the N-terminal of ApPCNA1. In the future, the effect of N-terminus deletion and binding of the DNA duplex on ApPCNA1 structure should be evaluated.

1) T. Yamauchi, et al., Purification and Crystallization of PCNA from thermophilic archaea. Poster presented at: 138th Annual Meeting of the Pharmaceutical Society of Japan; Mar. 25-28, 2018; Kanazawa, JAPAN.in a similar manner as the PCNA rings of other organisms; however, [i]Ap[/i]PCNA1 was located such that it did not form a perfect ring-shaped structure. The scattering curves of the complex and those of the model edited trimeric ring were almost similar with minor differences.

[b]Discussion[/b] The crystal structure of [i]Ap[/i]PCNA2 showed that it exists as a tetrameric-ring. PCNA is a trimeric ring. Although this molecule, which forms a trimer, is unstable as a tetramer, the crystal structure forms a tetrameric-ring. Hydrogen bonds present in neighboring molecule, from Thr175 to Glu179 and Glu109 to Ile114, form a $\beta$-sheet. This flexible interaction suggests that the protein could be forming tetrameric-ring-shaped structure. The crystal structure of [i]Ap[/i]PCNA3 shows that the $\mathrm{C}$-terminal contacts the cleavage site surrounded by $\beta$-sheets, formed behind the $\alpha$-helices, and the inter-domain connecting loop between the neighboring trimeric-rings. Leu249 in the C-terminal is placed in the hydrophobic pocket formed by Met40, Leu47, Leu131, Pro228, Ala246, and Pro247 (Figure). Thus, this hydrophobic cleavage site may be a binding site for PIP-box motif containing proteins. The N-terminus of [i]Ap[/i]PCNA1 is approximately 10 residues longer than that of [i]Ap[/i]PCNA2 and [i]Ap[/i]PCNA3. This could be why the tripartite complex is not ring shaped. Moreover, Met16 is present downstream of the N-terminal of [i]Ap[/i]PCNA1. In the future, the effect of N-terminus deletion and binding of the DNA duplex on [i]Ap[/i]PCNA1 structure should be evaluated.

1) T. Yamauchi, [i]et al.[/i], Purification and Crystallization of PCNA from thermophilic archaea. Poster presented at: 138[sup]th[/sup] Annual Meeting of the Pharmaceutical Society of Japan; Mar. 25-28, 2018; Kanazawa, JAPAN.

Acta Cryst. (2020). A76, a178-a179 


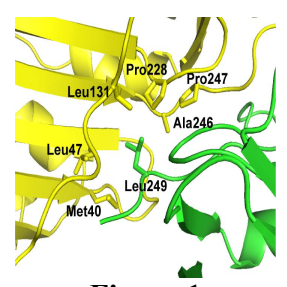

Figure 1

Acta Cryst. (2020). A76, a178-a179 